\title{
Distinctive collider signals for a two-Higgs-triplet model
}

\author{
Dilip Kumar Ghosh, ${ }^{1, *}$ Nivedita Ghosh, ${ }^{1, \dagger}$ and Biswarup Mukhopadhyaya ${ }^{2, *}$ \\ ${ }^{1}$ School Of Physical Sciences, Indian Association for the Cultivation of Science, \\ $2 A \& 2 B$, Raja S.C. Mullick Road, Kolkata 700032, India \\ ${ }^{2}$ Regional Centre for Accelerator-based Particle Physics, Harish-Chandra Research Institute, \\ Chhatnag Road, Jhunsi, Allahabad 211 019, India
}

(Received 13 August 2018; published 28 January 2019)

\begin{abstract}
The extension of the standard model (SM) with two complex $S U(2)_{L}$ scalar triplets enables one to have the Type II seesaw mechanism operative consistently with texture-zero neutrino mass matrices. This framework predicts additional doubly charged, singly charged and neutral spinless states. We show that, for certain values of the model parameters, there is sufficient mass splitting between the two doubly charged states $\left(H_{1}^{ \pm \pm}, H_{2}^{ \pm \pm}\right)$that allows the decay $H_{1}^{ \pm \pm} \rightarrow H_{2}^{ \pm \pm} h$, and thus leads to a unique signature of this scenario. We show that the final state $2\left(\ell^{ \pm} \ell^{ \pm}\right)+4 b+\mathbb{E}_{T}$ arising from this mode can be observed at the high energy, high luminosity (HE-HL) run of the $14 \mathrm{TeV}$ Large Hadron Collider (LHC), and also at a 100 TeV Future Circular Collider (FCC-hh).
\end{abstract}

DOI: 10.1103/PhysRevD.99.015036

\section{INTRODUCTION}

A $125 \mathrm{GeV}$ scalar, with a striking resemblance to the Higgs boson proposed in the standard model (SM) has been observed at the Large Hadron Collider (LHC) [1,2]. In spite of being a very successful phenomenological theory, the SM, however, cannot generate neutrino masses as suggested in various observations [3-5]. A popular set of mechanisms for generating such masses are the three types of seesaw mechanism [6-15]. Their experimental confirmation, on accelerators in particular are also of considerable interest [16-21]. Out of the three suggested types of seesaw, Type II involves an extension of the scalar sector with an additional complex $S U(2)_{L}$ triplet scalar with hypercharge $Y=2$. This triplet couples to leptons via interactions which violate lepton number by two units [9-13,22,23] and thus generates Majorana masses for neutrinos.

The most striking phenomenological consequence of such a triplet scalar is the presence of a doubly charged scalar. The triplet vacuum expectation value (vev), denoted here by $w$, is rather tightly constrained by the $\rho$-parameter to values less than about $5 \mathrm{GeV}$ [24]. The doubly charged

\footnotetext{
*tpdkg@iacs.res.in

Corresponding author.

tpng@iacs.res.in

*biswarup@hri.res.in
}

Published by the American Physical Society under the terms of the Creative Commons Attribution 4.0 International license. Further distribution of this work must maintain attribution to the author(s) and the published article's title, journal citation, and DOI. Funded by SCOAP ${ }^{3}$. scalar can decay either to produce same-sign dilepton peaks for $w<10^{-4} \mathrm{GeV}$, or to same-sign $W$-pairs for $w>$ $10^{-4} \mathrm{GeV}$ [25-27]. A rather strong lower limit of 770$800 \mathrm{GeV}$ exists on the doubly charged scalar mass in the former case, from same-sign dilepton searches at the LHC [28]. There is no such bound yet on doubly charged scalar mass for $w>10^{-4} \mathrm{GeV}$ [29]. This is because (a) a relatively large triplet vev implies small $\Delta L=2$ Yukawa couplings from a consideration of neutrino masses, and (b) overcoming standard model (SM) backgrounds for the final state driven by same-sign W-pairs is a challenging task, and requires a large integrated luminosity. Several works can be found in the literature, dwelling on strategies for unraveling the triplet scenario, both before [18-21, $30-32$ ] and after [33-42] the discovery of the $125 \mathrm{GeV}$ scalar.

From some special angles, however, a single triplet is inadequate for consistent neutrino mass generation in the Type-II seesaw model. For example, the somewhat different mass and mixing patterns in the neutrino sector (as compared to those in the quark sector) calls for studies in neutrino mass matrix models. One class of such models consists of zero textures, having some vanishing entries in the mass matrix, thus leading to relations between mass eigenvalues and mixing angles, and ensuring better predictiveness in the neutrino sector. It has been shown, that zero textures are inconsistent with Type-II seesaw models with a single scalar triplet [43]. Such inconsistency is removed when one has two such triplets, as has been demonstrated in [44]. This of course opens up the possibility of new collider signals which has been only partially investigated. 
In this work, we study the LHC signals that can decidedly tell us about the existence of two complex triplet scalars $\left(\Delta_{1}, \Delta_{2}\right)$. For example, in [44] searches via $\Delta_{1}^{ \pm \pm} \rightarrow$ $\Delta_{2}^{ \pm} W^{ \pm}$decay mode have been discussed. It should be noted that such a decay is disfavored in the single-triplet scenario, since the $\rho$-parameter restricts the mass splitting among fields having different electric charges. Another decay channel that opens up in this scenario is $\Delta_{1}^{ \pm \pm} \rightarrow \Delta_{2}^{ \pm \pm} h$, $\mathrm{h}$ being the SM-like Higgs. As has been shown in [45], this mode prevails especially in the presence of at least one $C P$-violating phase.

We neglect $C P$-violating phases in the present study. Regions of the parameter space answering to both $\Delta_{1}^{ \pm \pm} \rightarrow$ $\Delta_{2}^{ \pm} W^{ \pm}$and $\Delta_{1}^{ \pm \pm} \rightarrow \Delta_{2}^{ \pm \pm} h$ have been identified, and the corresponding signals have been predicted. Both these channels can lead to the final state $2 \ell^{ \pm} \ell^{ \pm}+4 b+\mathbb{E}_{T}$, where, $\ell \equiv e, \mu$. We carry out a detailed analysis to estimate signal significance of this scenario in the regions of the parameter space, consistent with all current limits both at the high energy high-luminosity (HE-HL) run of the LHC with $\sqrt{s}=14 \mathrm{TeV}$ and at the proposed $\sqrt{s}=$ $100 \mathrm{TeV}$ Future Circular Collider (FCC-hh) at CERN [46] or the Super Proton-Proton Collider (SPPC) in China [47]. Further details on the physics potential of the $100 \mathrm{TeV}$ collider can be found, for example, in [48]. We also comment on how to differentiate the two-triplet scenario from a single-triplet one using the signal analysis in this work.

The paper is organized as follows. In Sec. II we discuss a little bit about the well-motivated $Y=2$ single triplet scenario. Relevant phenomenological features of the twotriplet scenario are presented in Sec. III. Results of the collider analysis are reported in Sec. IV. We summarize and conclude in Sec. V.

\section{THE $Y=2$ SINGLE TRIPLET SCENARIO}

In this section we briefly describe the single triplet scenario. Along with the SM fields, there is an extra $S U(2)_{L}$ complex triplet scalar field $\Delta$ with hypercharge $Y=2$.

$$
\Delta=\frac{\sigma^{i}}{\sqrt{2}} \Delta_{i}=\left(\begin{array}{cc}
\delta^{+} / \sqrt{2} & \delta^{++} \\
\delta^{0} & -\delta^{+} / \sqrt{2}
\end{array}\right),
$$

where $\Delta_{1}=\left(\delta^{++}+\delta^{0}\right) / \sqrt{2}, \Delta_{2}=i\left(\delta^{++}-\delta^{0}\right) / \sqrt{2}, \Delta_{3}=\delta^{+}$.

The vevs of the doublet and the triplet are given by

$$
\langle\phi\rangle_{0}=\frac{1}{\sqrt{2}}\left(\begin{array}{l}
0 \\
v
\end{array}\right) \quad \text { and }\langle\Delta\rangle_{0}=\left(\begin{array}{cc}
0 & 0 \\
w & 0
\end{array}\right) \text {, }
$$

respectively and the electroweak vev is given by $v=$ $\sqrt{v^{2}+2 w^{2}}=246 \mathrm{GeV}$.
The most general scalar potential involving $\phi$ and $\Delta$ can be written as

$$
\begin{aligned}
V(\phi, \Delta)= & a \phi^{\dagger} \phi+\frac{b}{2} \operatorname{tr}\left(\Delta^{\dagger} \Delta\right)+c\left(\phi^{\dagger} \phi\right)^{2}+\frac{d}{4}\left(\operatorname{tr}\left(\Delta^{\dagger} \Delta\right)\right)^{2} \\
& +\frac{r}{2} \phi^{\dagger} \phi \operatorname{tr}\left(\Delta^{\dagger} \Delta\right)+\frac{f}{4} \operatorname{tr}\left(\Delta^{\dagger} \Delta^{\dagger}\right) \operatorname{tr}(\Delta \Delta) \\
& +h \phi^{\dagger} \Delta^{\dagger} \Delta \phi+\left(t \phi^{\dagger} \Delta \tilde{\phi}+\text { H.c. }\right),
\end{aligned}
$$

where $\tilde{\phi} \equiv i \tau_{2} \phi^{*}$. In general, both $v$ and $w$ can be complex. However, since we want to avoid all $C P$-violating effects, we choose both the vevs to be real and positive, which as a result implies that $t$ has to be real.

It should be remembered that the choice $a<0, b>0$ ensures the primary source of spontaneous symmetry breaking to be the vev of the scalar doublet. At the same time, the $\rho$-parameter has to be very close to its tree-level SM value of unity, as required by the latest data, namely, $\rho=1.0004_{-0.0004}^{+0.0003}$ [49] for $w \ll v$. Also the doublet-triplet mixing has to be small and the perturbativity of all quartic couplings at the electroweak scale has to be guaranteed.

All the aforementioned constraints drive us to choose the following orders of magnitude for the parameters in the potential:

$$
a, b \sim v^{2} ; \quad c, d, r, f, h \sim 1 ; \quad|t| \ll v .
$$

The mass terms for singly-charged scalars in this model are given by

$$
\mathcal{L}_{S}^{ \pm}=-\left(H^{-}, \phi^{-}\right) \mathcal{M}_{+}^{2}\left(\begin{array}{c}
H^{+} \\
\phi^{+}
\end{array}\right)
$$

where

$\mathcal{M}_{+}^{2}=\left(\begin{array}{cc}(q+h / 2) v^{2} & \sqrt{2} v(t-w h / 2) \\ \sqrt{2} v(t-w h / 2) & 2(q+h / 2) w^{2}\end{array}\right)$ and $q=\frac{|t|}{w}$.

Diagonalization of the matrix should yield one zero eigenvalue, corresponding to the Goldstone boson. The singly-charged mass-squared matrix is

$$
m_{\Delta^{+}}^{2}=\left(q+\frac{h}{2}\right)\left(v^{2}+2 w^{2}\right),
$$

whereas the doubly-charged scalar mass is

$$
m_{\Delta^{++}}^{2}=(h+q) v^{2}+2 f w^{2} .
$$

In the limit $w \ll v$, one obtains

$$
m_{\Delta^{++}}^{2}-m_{\Delta^{+}}^{2} \simeq \frac{h}{2} v^{2} .
$$

Electroweak precision data imply $\Delta M \equiv\left|m_{\Delta^{ \pm \pm}}-m_{\Delta^{ \pm}}\right| \lesssim$ $50 \mathrm{GeV}[50,51]$ assuming a light SM Higgs boson of 
mass $m_{h}=125 \mathrm{GeV}$ and top quark mass $M_{t}=173 \mathrm{GeV}$. Hence, the decay mode $\Delta^{++} \rightarrow \Delta^{+} W^{+}$is kinematically not allowed with a single triplet scalar.

\section{EXTENSION WITH TWO TRIPLETS}

The single-triplet scenario can sometimes turn out to be inadequate. For example, the somewhat novel kind of bilarge mixing in the neutrino sector motivates people to link such a mixing pattern with the neutrino mass matrix itself. The number of arbitrary parameters in such an investigation is reduced, and the mass eigenvalues and mixing angles are linked in a predictive manner, if some elements of this matrix vanish. It is with this in view that various texture zero neutrino mass matrices have been proposed, for example, through the imposition of certain Abelian symmetries. Two-zero textures constitute a popular subset of such models, which have been widely used in various contexts [43,52-65].

In the specific context of Type II seesaw, however, inconsistencies arise when texture zeros (especially twozero textures) are attempted [66]. Such inconsistency can be avoided, as already mentioned, when two scalar triplets are present. In such a scenario, one extends the SM with two $Y=2$ triplet scalars $\Delta_{1}, \Delta_{2}$ :

$$
\Delta_{1}=\left(\begin{array}{cc}
\delta_{1}^{+} & \sqrt{2} \delta_{1}^{++} \\
\sqrt{2} \delta_{1}^{0} & -\delta_{1}^{+}
\end{array}\right) \text {and } \Delta_{2}=\left(\begin{array}{cc}
\delta_{2}^{+} & \sqrt{2} \delta_{2}^{++} \\
\sqrt{2} \delta_{2}^{0} & -\delta_{2}^{+}
\end{array}\right)
$$

The vevs of the scalar triplets are given by

$$
\left\langle\Delta_{1}\right\rangle_{0}=\left(\begin{array}{cc}
0 & 0 \\
w_{1} & 0
\end{array}\right) \quad \text { and } \quad\left\langle\Delta_{2}\right\rangle_{0}=\left(\begin{array}{cc}
0 & 0 \\
w_{2} & 0
\end{array}\right)
$$

The scalar potential in this scenario involving the Higgs doublet and the two triplets can be written as

$$
\begin{aligned}
V\left(\phi, \Delta_{1}, \Delta_{2}\right)= & a \phi^{\dagger} \phi+\frac{1}{2} b_{k l} \operatorname{tr}\left(\Delta_{k}^{\dagger} \Delta_{l}\right)+c\left(\phi^{\dagger} \phi\right)^{2} \\
& +\frac{1}{4} d_{k l}\left(\operatorname{tr}\left(\Delta_{k}^{\dagger} \Delta_{l}\right)\right)^{2}+\frac{1}{2} r_{k l} \phi^{\dagger} \phi \operatorname{tr}\left(\Delta_{k}^{\dagger} \Delta_{l}\right) \\
& +\frac{1}{4} f_{k l} \operatorname{tr}\left(\Delta_{k}^{\dagger} \Delta_{l}^{\dagger}\right) \operatorname{tr}\left(\Delta_{k} \Delta_{l}\right)+h_{k l} \phi^{\dagger} \Delta_{k}^{\dagger} \Delta_{l} \phi \\
& +g \operatorname{tr}\left(\Delta_{1}^{\dagger} \Delta_{2}\right) \operatorname{tr}\left(\Delta_{2}^{\dagger} \Delta_{1}\right)+g^{\prime} \operatorname{tr}\left(\Delta_{1}^{\dagger} \Delta_{1}\right) \operatorname{tr}\left(\Delta_{2}^{\dagger} \Delta_{2}\right) \\
& +\left(t_{k} \phi^{\dagger} \Delta_{k} \widetilde{\phi}+\text { H.c. }\right),
\end{aligned}
$$

where $k, l=1,2$.

As mentioned in the previous section, $v, w_{1}, w_{2}$ as well as $t_{1}, t_{2}$ are taken to be real and positive. One can also use for illustration, without any loss in the generality of the results, $a, b_{k l} \sim v^{2} ; \quad c, d_{k l}, r_{k l}, h_{k l}, f_{k l}, g, g^{\prime} \sim \mathcal{O}(1) ; \quad\left|t_{k}\right| \ll v$.

With $w_{1}, w_{2} \ll v$.

We redefine the following $2 \times 2$ matrices and vectors:

$$
\begin{aligned}
B & =\left(b_{k l}\right), \quad E=\left(r_{k l}+h_{k l}\right), \quad H=\left(h_{k l}\right), \\
t & =\left(\begin{array}{c}
t_{1} \\
t_{2}
\end{array}\right), \quad w=\left(\begin{array}{l}
w_{1} \\
w_{2}
\end{array}\right) .
\end{aligned}
$$

The minimization of the potential (12), neglecting all terms quartic in the triplet vevs, yields

$$
\begin{gathered}
\left(B+\frac{v^{2}}{2}(E-H)\right) w+v^{2} t=0, \\
a+c v^{2}+\frac{1}{2} w^{T}(E-H) w+2 t \cdot w=0,
\end{gathered}
$$

where we have used $t \cdot w=\sum_{k} t_{k} w_{k}$. Solving Eqs. (15) and (16) simultaneously, $w_{k}(k=1,2)$ are obtained as

$$
w=-v^{2}\left(B+\frac{1}{2} v^{2}(E-H)\right)^{-1} t .
$$

After diagoalization of different kinds of scalar mass matrices following electroweak symmetry breaking (EWSB), we obtain the charged scalars $\left(H_{1}^{ \pm \pm}, H_{2}^{ \pm \pm}\right)$, singly charged $\operatorname{Higgs}\left(H_{1}^{ \pm}, H_{2}^{ \pm}\right)$, and the neutral $C P$-even $\left(h, H_{1}, H_{2}\right)$ and $C P$-odd $\left(A_{1}, A_{2}\right)$ scalars. Among them $h$ is the SM-like Higgs.

The mass matrix of the doubly-charged scalars is given by

$$
\mathcal{M}_{ \pm \pm}^{2}=B+\frac{v^{2}}{2}(E+H) .
$$

which can be diagonalized by

$$
U^{\dagger} \mathcal{M}_{ \pm \pm}^{2} U=\operatorname{diag}\left(M_{1}^{2}, M_{2}^{2}\right)
$$

yielding the mass eigenstates:

$$
\left(\begin{array}{l}
\delta_{1}^{ \pm \pm} \\
\delta_{2}^{ \pm \pm}
\end{array}\right)=U\left(\begin{array}{l}
H_{1}^{ \pm \pm} \\
H_{2}^{ \pm \pm}
\end{array}\right)
$$
from

$$
-\mathcal{L}_{S}^{ \pm}=\left(\delta_{1}^{-}, \delta_{2}^{-}, \phi^{-}\right) \mathcal{M}_{ \pm}^{2}\left(\begin{array}{c}
\delta_{1}^{+} \\
\delta_{2}^{+} \\
\phi^{+}
\end{array}\right)+\text {H.c. }
$$


where

$$
\mathcal{M}_{ \pm}^{2}=\left(\begin{array}{cc}
B+\frac{v^{2}}{2} E & \sqrt{2} v(t-H w / 2) \\
\sqrt{2} v(t-H w / 2)^{\dagger} & a+c v^{2}+\frac{1}{2} w^{T}(E+H) w
\end{array}\right) .
$$

Using Eqs. (15) and (16) we get,

$$
\mathcal{M}_{ \pm}^{2}\left(\begin{array}{c}
v_{T} \\
v / \sqrt{2}
\end{array}\right)=0
$$

This serves as a consistency check that the singly charged mass matrix has to have an eigenvector with zero eigenvalue that corresponds to the would-be-Goldstone boson.

It is evident from Eq. (13) that $\mathrm{b}$ is of the order of $v^{2}$. Therefore, in a rough approximation one can safely ignore the $t_{k}$ and the triplet vevs in the mass matrix $\mathcal{M}_{ \pm}^{2}$. In that limit, also $a+c v^{2}=0$ and the charged would-beGoldstone boson is equivalent to $\phi^{ \pm}$. There is no mixing with the $\delta_{k}^{ \pm}$.

The singly charged mass matrix can be diagonalized by

$$
V^{\dagger} \mathcal{M}_{ \pm}^{2} V=\operatorname{diag}\left(\mu_{1}^{2}, \mu_{2}^{2}, 0\right),
$$

with

$$
\left(\begin{array}{c}
\delta_{1}^{ \pm} \\
\delta_{2}^{ \pm} \\
\phi^{+}
\end{array}\right)=V\left(\begin{array}{c}
H_{1}^{ \pm} \\
H_{2}^{ \pm} \\
G^{ \pm}
\end{array}\right)
$$

Where $G^{ \pm}$is the charged would-be-Goldstone boson.

Interactions with the W-boson are given by

$$
\begin{aligned}
\mathcal{L}_{\text {gauge }}= & i g \sum_{k=1}^{2}\left[\delta_{k}^{-}\left(\partial^{\mu} \delta_{k}^{++}\right)-\left(\partial^{\mu} \delta_{k}^{-}\right) \delta_{k}^{++}\right] W_{\mu}^{-} \\
& -\frac{g^{2}}{\sqrt{2}} \sum_{k=1}^{2} w_{k} W_{\mu}^{-} W^{-\mu} \delta_{k}^{++}+\text {H.c. }
\end{aligned}
$$

$$
U=\left(\begin{array}{c}
c_{12} c_{13} \\
-s_{12} c_{23}-c_{12} s_{23} s_{13} e^{i \delta} \\
s_{12} s_{23}-c_{12} c_{23} s_{13} e^{i \delta}
\end{array}\right.
$$

and $\hat{M}_{\nu}$ is the diagonal matrix of the neutrino masses. We have dropped possible Majorana phases for simplicity. Global analyses of data can be used to resolve the various

\footnotetext{
${ }^{1} \mathrm{We}$ assume the charged-lepton mass matrix to be already diagonal.
}

Here $g$ is the $S U(2)_{L}$ gauge coupling constant. Changing the gauge basis into mass basis allows us to compute the decay rates of $\mathrm{H}_{1}^{++} \rightarrow \mathrm{H}_{2}^{+} \mathrm{W}^{+}$and $\mathrm{H}_{k}^{++} \rightarrow W^{+} W^{+}$ $(k=1,2)$.

The $(\Delta L=2)$ Yukawa interaction Lagrangian involving the triplets and the leptons is

$$
\mathcal{L}_{Y}=\frac{1}{2} \sum_{k=1}^{2} h_{i j}^{(k)} L_{i}^{T} C^{-1} i \tau_{2} \Delta_{k} L_{j}+\text { H.c. },
$$

where $L_{i}$ denote the left-handed lepton doublets, $C$ is the Dirac charge conjugation matrix, the $h_{i j}^{(k)}$ are the symmetric neutrino Yukawa coupling matrices of the triplets $\Delta_{k}$, and the $i, j=1,2,3$ are the summation indices over the three neutrino flavours. ${ }^{1}$

When the triplets acquire vev from Eq. (27) one can generate the neutrino mass matrix as:

$$
\left(M_{\nu}\right)_{i j}=h_{i j}^{(1)} w_{1}+h_{i j}^{(2)} w_{2}
$$

This connects the Yukawa coupling constants $h_{i j}^{(1)}, h_{i j}^{(2)}$ and the triplet vevs $w_{1}, w_{2}$.

In this work we use, once more as illustration, the normal hierarchy of the neutrino mass spectrum and set the lowest neutrino mass eigenvalue to zero. The elements of the neutrino mass matrix $M_{\nu}$ can be obtained by using the observed central values of the various lepton mixing angles and by diagonalizing as

$$
M_{\nu}=U \hat{M}_{\nu} U^{\dagger}
$$

where $U$ is the Pontecorvo-Maki-Nakagawa-Sakata matrix given by [67]

$$
\left.\begin{array}{cc}
s_{12} c_{13} & s_{13} e^{-i \delta} \\
c_{12} c_{23}-s_{12} s_{23} s_{13} e^{i \delta} & s_{23} c_{13} \\
-c_{12} s_{23}-s_{12} c_{23} s_{13} e^{i \delta} & c_{23} c_{13}
\end{array}\right)
$$

entries of $U$ [68]. The left-hand side of Eq. (28) is reliably represented, at least in orders of magnitude, by the central values of all angles, including that for $\theta_{13}$ as obtained from the recent Daya Bay and RENO experiments [69,70]. The actual mass matrix thus constructed has some elements at least one order of magnitude smaller than the others, thus suggesting texture zeros. 
TABLE I. Parameters for all benchmark points.

\begin{tabular}{|c|c|c|c|c|c|c|c|c|c|c|c|c|c|c|c|c|c|}
\hline $\mathrm{a}$ & & B & I & D & E & E & I & & I & 4 & c & $\mathrm{g}$ & $g^{\prime}$ & $t_{1}$ & $t_{2}$ & $\begin{array}{c}w_{1} \\
(\text { in } \mathrm{GeV})\end{array}$ & (in $\left.\begin{array}{c}w_{2} \\
\mathrm{GeV}\end{array}\right)$ \\
\hline BP1 -15625 & $\begin{array}{c}41012.0 \\
-31980.0\end{array}$ & $\left.\begin{array}{c}-31980.0 \\
21182.4\end{array}\right)$ & $\left(\begin{array}{l}4.00 \\
3.56\end{array}\right.$ & $\left.\begin{array}{l}3.56 \\
4.00\end{array}\right)$ & $\left(\begin{array}{l}2.64 \\
2.80\end{array}\right.$ & $\left.\begin{array}{l}2.80 \\
2.64\end{array}\right)$ & $\left(\begin{array}{l}1.00 \\
1.00\end{array}\right.$ & $\left.\begin{array}{l}1.00 \\
1.00\end{array}\right)$ & $\left(\begin{array}{l}4.00 \\
2.00\end{array}\right.$ & $\left.\begin{array}{l}2.00 \\
4.00\end{array}\right)$ & 0.26 & 0.90 & 0.90 & -1 & -2 & 1.09 & 1.32 \\
\hline BP2 -15625 & $\left(\begin{array}{c}43012.0 \\
-31998.0\end{array}\right.$ & $\left.\begin{array}{c}-31998.0 \\
23182.4\end{array}\right)$ & $\left(\begin{array}{l}4.00 \\
3.40\end{array}\right.$ & $\left.\begin{array}{l}3.40 \\
4.00\end{array}\right)$ & $\left(\begin{array}{l}2.54 \\
2.80\end{array}\right.$ & $\left.\begin{array}{l}2.80 \\
2.54\end{array}\right)$ & $\left(\begin{array}{l}1.00 \\
1.00\end{array}\right.$ & $\left.\begin{array}{l}1.00 \\
1.00\end{array}\right)$ & $\left(\begin{array}{l}3.50 \\
2.00\end{array}\right.$ & $\left.\begin{array}{l}2.00 \\
3.50\end{array}\right)$ & 0.24 & 0.90 & 0.90 & -1 & -2 & 1.09 & 1.32 \\
\hline BP3 -15625 & $\left(\begin{array}{c}41012.0 \\
-31998.0\end{array}\right.$ & $\left.\begin{array}{c}-31998.0 \\
21182.4\end{array}\right)$ & $\left(\begin{array}{l}4.00 \\
3.60\end{array}\right.$ & $\left.\begin{array}{l}3.60 \\
4.00\end{array}\right)$ & $\left(\begin{array}{l}2.74 \\
2.80\end{array}\right.$ & $\left.\begin{array}{l}2.80 \\
2.74\end{array}\right)$ & $\left(\begin{array}{l}1.00 \\
1.00\end{array}\right.$ & $\left.\begin{array}{l}1.00 \\
1.00\end{array}\right)$ & $\left(\begin{array}{l}3.00 \\
2.00\end{array}\right.$ & $\left.\begin{array}{l}2.00 \\
3.00\end{array}\right)$ & 0.25 & 0.90 & 0.90 & -1 & -2 & 1.09 & 1.32 \\
\hline
\end{tabular}

\section{ANALYSIS}

Let us now look for smoking gun collider signals of doubly charged scalars of this scenario with $w_{k} \sim$ $\mathcal{O}(1) \mathrm{GeV}$. The spectacular $l^{ \pm} l^{ \pm}$decay channels are suppressed in this case. The doubly charged scalars now mainly decay into the following final states:

$$
\begin{aligned}
& H_{1}^{ \pm \pm} \rightarrow H_{2}^{ \pm \pm} h, \\
& H_{1}^{ \pm \pm} \rightarrow W^{ \pm} W^{ \pm}, \\
& H_{1}^{ \pm \pm} \rightarrow H_{2}^{ \pm} W^{ \pm}, \\
& H_{2}^{ \pm \pm} \rightarrow W^{ \pm} W^{ \pm} .
\end{aligned}
$$

The decay mode as mentioned in Eq. (31) is absent in the single-triplet model, since there is only one doubly charged scalar particle. Also, the equivalent of Eq. (33), namely, $H_{1}^{ \pm \pm} \rightarrow H_{1}^{ \pm} W^{ \pm}$is kinematically disfavored since the mass splitting between singly and doubly charged scalar is restricted by the $\rho$ parameter constraint [49-51]. Hence, in order to distinguish between the single triplet and the double triplet scalar model, it is advantageous to investigate channels in Eqs. (31) and (33), since the corresponding event topologies cannot be faked by a single-triplet scenario. The production of $W^{ \pm}$in association with the SM-like Higgs boson leads to the following final state:

$$
2 \ell^{ \pm} \ell^{ \pm}+4 b+\mathbb{E}_{T}
$$

where $\ell=e, \mu$, which arise from $W^{ \pm} \rightarrow \ell^{ \pm} \nu_{\ell}\left(\bar{\nu}_{\ell}\right)$ and $h \rightarrow b \bar{b}$ decay modes.

Since the doublet-triplet mixing is small in this model, there is no noticeable difference in production rate in the gluon fusion channel and also the tree-level decay of the SM-like Higgs. However, the presence of $H_{1}^{ \pm \pm}, H_{2}^{ \pm \pm}$and $H_{1}^{ \pm}, H_{2}^{ \pm}$modify the loop-induced $h \rightarrow \gamma \gamma$ decay significantly. Detailed analyses of such modification can be found in $[24,50,71-75]$. Here we just ensure that our benchmark points are consistent with the limits on the diphoton signal strength of the Higgs at the $2 \sigma$ level [76].

\section{A. Benchmark points}

For collider analysis we choose three representative benchmark points such that the decays $\mathrm{H}_{1}^{++} \rightarrow \mathrm{H}_{2}^{++} h$ and $\mathrm{H}_{1}^{++} \rightarrow \mathrm{H}_{2}^{+} \mathrm{W}^{+}$are kinematically allowed. Also, the mass splitting $\Delta M$ between the doubly and singly charged Higgses for each of the triplet scalar fields are consistent $(\Delta M \leq 50 \mathrm{GeV})[50,51]$ with the $\rho$ parameter constraint. All three benchmark points are also consistent with the observed Higgs signal strength. In Table I we show thirteen model parameters as defined in the scalar potential in Eq. (12) that determine the benchmark points. One can see from the table that the values of some of the parameters are kept fixed while others $(B, D, E, F, c)$ have been varied, since the scalar masses are strongly dependent on the latter. ${ }^{2}$ Table II lists the corresponding values of neutral, singly charged and doubly charged scalar masses. The corresponding mixing angles in different sectors are shown in Table III. The mixing angles between the doubly charged higgses, singly charged scalars, and $C P$ even neutral scalars (same as $C P$ odd scalars) are denoted by $\alpha, \beta$ and $\gamma$ respectively.

Even after fixing fixed all model parameters in Table I, the Yukawa coupling matrices $h^{(1)}$ and $h^{(2)}$ still remain indeterminate [Eq. (28)]. We fix the matrix $h^{(2)}$ by choosing one suitable value for all elements of the $\mu-\tau$ block and keeping the rest of the elements one order smaller. It is emphasized that this ad hoc convention does not affect the generality of our results. One may thus write

$$
\begin{aligned}
& h_{i j}^{(1)}=\left(\begin{array}{lll}
4.52 \times 10^{-12} & 1.02 \times 10^{-11} & 3.47 \times 10^{-12} \\
1.02 \times 10^{-11} & 2.12 \times 10^{-11} & 1.90 \times 10^{-11} \\
3.47 \times 10^{-12} & 1.90 \times 10^{-11} & 3.68 \times 10^{-11}
\end{array}\right), \\
& h_{i j}^{(2)}=\left(\begin{array}{lll}
1.0 \times 10^{-12} & 1.0 \times 10^{-12} & 1.0 \times 10^{-12} \\
1.0 \times 10^{-12} & 1.0 \times 10^{-11} & 1.0 \times 10^{-11} \\
1.0 \times 10^{-12} & 1.0 \times 10^{-11} & 1.0 \times 10^{-11}
\end{array}\right) .
\end{aligned}
$$

\footnotetext{
${ }^{2}$ We have kept the triplet vevs fixed at $\sim 1 \mathrm{GeV}$ throughout our analysis to allow maximum mixing between the doublet and the triplet scalars as permitted by $\rho$ parameter constraint [24] for all the three benchmark points noted in Table I. In principle, one can reduce the vevs up to $10^{-4} \mathrm{GeV}$ [25-27] and that will not change the decay modes of the nonstandard scalars.
} 
TABLE II. Masses for different nonstandard scalars and the corresponding SM Higgs signal strength within $2 \sigma$ limit of the current value $1.02_{-0.19}^{+0.18}[76]$.

\begin{tabular}{|c|c|c|c|c|c|c|c|}
\hline & $m_{H_{1}^{ \pm \pm}}($in $\mathrm{GeV})$ & $m_{H_{2}^{ \pm \pm}}($in $\mathrm{GeV})$ & $m_{H_{1}^{ \pm}}($in $\mathrm{GeV})$ & $m_{H_{2}^{ \pm}}($in $\mathrm{GeV})$ & $m_{H_{1}} / m_{A_{1}}($ in $\mathrm{GeV})$ & $m_{H_{2}} / m_{A_{2}}($ in $\mathrm{GeV})$ & $\mu_{\gamma \gamma}$ \\
\hline BP1 & 416.37 & 216.13 & 402.13 & 215.87 & 276.56 & 161.48 & 1.20 \\
\hline BP2 & 474.20 & 240.09 & 450.80 & 239.40 & 229.60 & 167.60 & 1.15 \\
\hline BP3 & 490.39 & 365.26 & 483.68 & 310.39 & 328.30 & 272.23 & 1.07 \\
\hline
\end{tabular}

TABLE III. Mixing angles between for the doubly charged $(\alpha)$, singly charged $(\beta)$ and $C P$-even $(C P$-odd) scalars $(\gamma)$.

\begin{tabular}{lccc}
\hline \hline & $\sin \alpha$ & $\sin \beta$ & $\sin \gamma$ \\
\hline BP1 & 0.76 & 0.63 & 0.55 \\
BP2 & 0.66 & 0.65 & 0.35 \\
BP3 & 0.73 & 0.70 & 0.61 \\
\hline \hline
\end{tabular}

\section{B. Collider search at the LHC}

We finally turn to signals of this scenario at the high energy high luminosity (HE-HL) run of the LHC. There are several production and decay chains that lead to our chosen final state Eq. $(35)^{3}$

$$
\begin{aligned}
p p & \rightarrow H_{1}^{++} H_{1}^{--} \rightarrow\left(H_{2}^{++} h\right)+\left(H_{2}^{--} h\right) \\
& \rightarrow\left(W^{+} W^{+} h\right)+\left(W^{-} W^{-} h\right) \rightarrow 2\left(\ell^{ \pm} \ell^{ \pm}\right)+4 b+\boldsymbol{E}_{T}
\end{aligned}
$$

$$
\begin{aligned}
p p & \rightarrow H_{1}^{++} H_{1}^{--} \rightarrow\left(H_{2}^{+} W^{+}\right)+\left(H_{2}^{-} W^{-}\right) \\
& \rightarrow\left(W^{+} W^{+} h\right)+\left(W^{-} W^{-} h\right) \rightarrow 2\left(\ell^{ \pm} \ell^{ \pm}\right)+4 b+\mathbb{E}_{T}
\end{aligned}
$$

$$
\begin{aligned}
p p & \rightarrow H_{1}^{++} H_{1}^{--} \rightarrow\left(H_{2}^{+} W^{+}\right)+\left(H_{2}^{--} h\right) \\
& \rightarrow\left(W^{+} W^{+} h\right)+\left(W^{-} W^{-} h\right) \rightarrow 2\left(\ell^{ \pm} \ell^{ \pm}\right)+4 b+\mathbb{E}_{T}
\end{aligned}
$$

$$
\begin{aligned}
p p & \rightarrow H_{1}^{++} H_{1}^{--} \rightarrow\left(H_{2}^{++} h\right)+\left(H_{2}^{-} W^{-}\right) \\
& \rightarrow\left(W^{+} W^{+} h\right)+\left(W^{-} W^{-} h\right) \rightarrow 2\left(\ell^{ \pm} \ell^{ \pm}\right)+4 b+\boldsymbol{E}_{T}
\end{aligned}
$$

It should be noted that $\operatorname{Br}\left(H_{2}^{+} \rightarrow W^{+} Z\right)$ and $\operatorname{Br}\left(H_{2}^{+} \rightarrow\right.$ $t \bar{b})$ could give rise to the same final state. However, $\operatorname{Br}\left(H_{2}^{+} \rightarrow W^{+} h\right)$ is $99.99 \%, 97.60 \%$ and $99.5 \%$ for $\mathrm{BP} 1$, $\mathrm{BP} 2$, and BP3 respectively. The $W^{+} h$ mode dominates over $W^{+} Z$ and $t \bar{b}$ as the $W^{+} h$ channel receives a single-fold suppression due to doublet-triplet mixings while the two

\footnotetext{
${ }^{3}$ For benchmark points BP1 and BP3, the main contribution comes from (36b) as $\mathrm{BR}\left(H_{1}^{++} \rightarrow H_{2}^{+} W^{+}\right) \simeq 99 \%$. However, situation is different for $\mathrm{BP} 2$, where, $\mathrm{BR}\left(H_{1}^{++} \rightarrow H_{2}^{++} h\right) \simeq$ $23 \%$, making all the four production and decay modes significant.
}

remaining channels $\left(W^{+} Z, t \bar{b}\right)$ have two-fold suppression, namely, that from mixing as well as by the small triplet vev which explicitly enters into the couplings. There is a further cancellation between the two above mentioned effects for the $W^{+} Z$ and $t \bar{b}$ channels making the branching fraction negligible for these two decay modes.

We calculate the event rates in MADGRAPH5(v2.4.3) [77] with the appropriate Feynman rules obtained via FEYNRULES [78]. The signal as well as all the relevant standard model background events are calculated at the lowest order (LO) with CTEQ6L [79] parton distribution functions, setting the renormalization and factorization scales at $M_{Z}$. They are subsequently multiplied by the next-to-leading order (NLO) K-factors for the signal and the SM background processes, taken as 1.25 [80] and 1.3 [81-83] respectively. For the showering and hadronization of both the signal and the SM background events we use the Pythia(v6.4) [84], and the detector simulation is done in DELPHES(v3) [85], where jets are constructed using the anti$K_{T}$ algorithm [86]. The cut-based analyses are done using the MadAnAlysis5 [87]. The production of $t \bar{t} Z, t \bar{t} W^{ \pm}$and $t \bar{t} h$ constitute the dominant SM background for our signal. While generating events, we select jets and leptons (electron and muon) using the following kinematical acceptance cuts:

$$
\begin{aligned}
& \Delta R_{j j}>0.6, \quad \Delta R_{\ell \ell}>0.4, \quad \Delta R_{j \ell}>0.7, \\
& \Delta R_{b j}>0.7, \quad \Delta R_{b \ell}>0.2, \\
& p_{T_{\min }}^{j}>20 \mathrm{GeV}, \quad\left|\eta_{j}\right|<5, \\
& p_{T_{\min }}^{\ell}>10 \mathrm{GeV}, \quad\left|\eta_{\ell}\right|<2.5,
\end{aligned}
$$

The presence of four b-jets in the signal makes b-tagging an important issue. For this we comply with the efficiency formula proposed by the ATLAS collaboration [88] for both the signal and background processes as follows:

$$
\epsilon_{b}= \begin{cases}0 & p_{T}^{b} \leq 30 \mathrm{GeV} \\ 0.6 & 30 \mathrm{GeV}<p_{T}^{b}<50 \mathrm{GeV} \\ 0.75 & 50 \mathrm{GeV}<p_{T}^{b}<400 \mathrm{GeV} \\ 0.5 & p_{T}^{b}>400 \mathrm{GeV}\end{cases}
$$



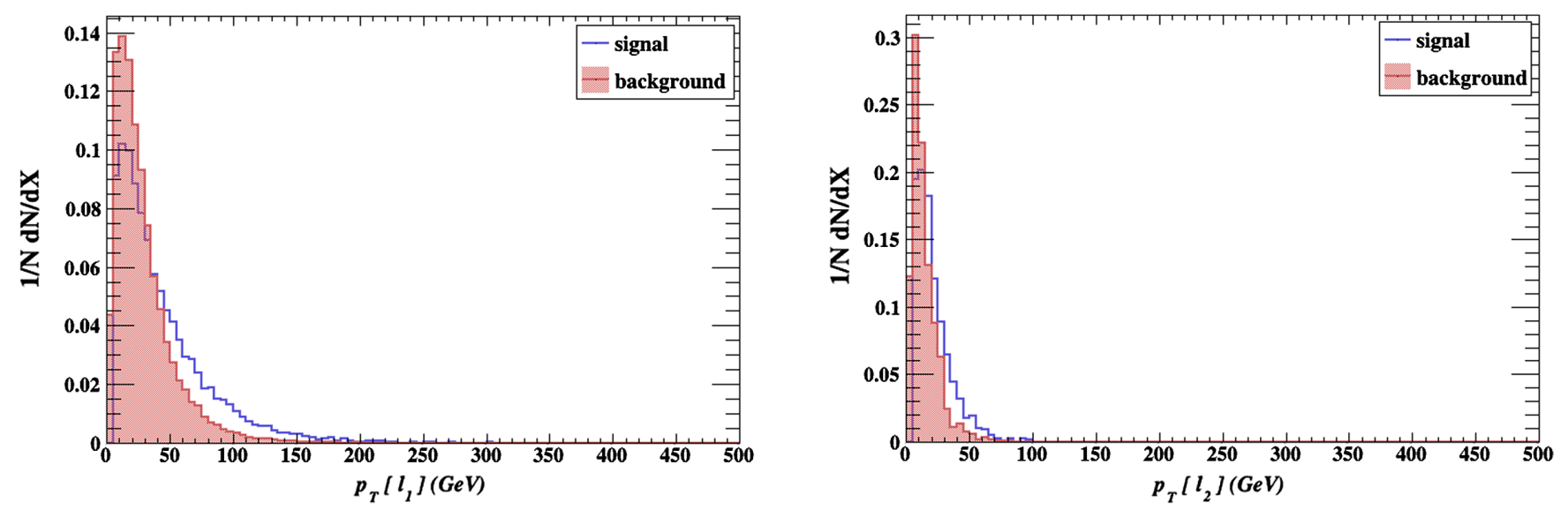

FIG. 1. Transverse momentum $\left(p_{T}\right)$ distribution (normalized) of the leading and subleading leptons for the benchmark BP1.

In addition, a mistagging probability of $10 \%(1 \%)$ for charm-jets (light-quark and gluon jets) as a $b$-jet has been taken into account. For lepton isolation, we abide by the criteria defined in Ref. [89] where the electrons are isolated with the Tight criterion defined in Ref. [90] and the muons are isolated using the Medium criterion defined in Ref. [91].

One point worth mentioning at this point is that we consider the all inclusive decay channels for both the signal and background event generation, not only the leptonic decay of the SM $W^{ \pm}$boson. This is true for all the subsequent analyses. Before reporting the results in detail, let us examine some kinematic distributions relevant for the analysis, starting with transverse momenta $\left(p_{T}\right)$ of the two leading leptons as depicted in Fig. 1 for BP1. The signal and background distributions are shown in blue and red respectively. In the signal events, these leptons originate from the decay of the $W$ boson, while for the SM background processes, they come from the decay of $W^{ \pm}$or Z-bosons. From the shape of the $p_{T}$ distribution one can see that it is evidently difficult to impose any selection cut on the $p_{T}$ of these two leading leptons to distinguish the signal from the SM backgrounds. This general feature is also found for other two benchmarks points. Hence, we only put the basic acceptance cut on the $p_{T}$ of the leptons, $p_{T}>10 \mathrm{GeV}$.

In Fig. 2 we show the $P_{T}$ distributions of four $b$-jets for both signal and background events. In the signal events, they come from decays of two SM-like scalars each of which is produced via either from $H_{1}^{ \pm \pm} \rightarrow H_{2}^{ \pm \pm} h \rightarrow W^{ \pm} W^{ \pm} h$ or from $H_{1}^{ \pm \pm} \rightarrow H_{2}^{ \pm} W^{ \pm} \rightarrow W^{ \pm} W^{ \pm} h$. On the other hand, the background $b$-jets have their sources mostly in $t \bar{t} h, t \bar{t} W^{ \pm}$ and $t \bar{t} Z$. In the case of $t \bar{t} W^{ \pm}$production, two $b$-jets come from the top quarks, while a pair of light quark jets may fake as $b$-jets. The leading $b$-jet of our signal events is found to be harder than that of those in the background. Thus we demand the leading $b$-jet to have $p_{T}\left(j_{1}\right)>80 \mathrm{GeV}$, and $p_{T}$ of the subsequent $3 b$-jets to be $p_{T}\left(j_{2}\right)>60 \mathrm{GeV}$, $p_{T}\left(j_{3}\right)>30 \mathrm{GeV}$, and $p_{T}\left(j_{4}\right)>20 \mathrm{GeV}$.
The normalized distribution of the missing transverse energy $\left(\boldsymbol{E}_{T}\right)$ are in the left panel of Fig. 3. One should note that the $\mathbb{E}_{T}$ for both signal and backgrounds is due to either neutrinos or the mismeasurement of the jet and lepton momenta. Consequently, the shape of the distributions for both the signal and the background look very similar. The small rightward shift of the peak of the signal distribution can be attributed to fact that $W^{ \pm}$are boosted as they are produced from the decays of much heavier parent scalars. Hence, we find that a moderate requirement of $\mathbb{E}_{T}>$ $30 \mathrm{GeV}$ is sufficient to improve the signal to background ratio.

The right panel of Fig. 3 shows the normalized distribution of angular separation $\Delta R\left(\ell^{ \pm} \ell^{ \pm}\right)$between two samesign leptons for both the signal and the background events. For our signal events, both leptons come from same-sign $W^{ \pm}$which are produced from the decay of heavy doubly charged scalar with nonzero transverse momenta. The $P_{T}$ of the heavy doubly charged scalar forces its decay products ( $W$-pair) to appear with relatively small $\Delta R$. The resultant effect of these two is finally displayed by the two same-sign leptons that also appear with small opening angle. For the SM background, on the other hand, they come from $W^{ \pm} / Z$ bosons radiated from top/antitop quarks, and can have wider separations. An upper cut on $\Delta R\left(\ell_{1}^{ \pm} \ell_{2}^{ \pm}\right)<1.5$ thus enhances our signal-to-background ratio.

The cuts are summarized below, in the order in which they are imposed:

(i) $(C-1)$ : We want the leading $b$-jet to have $p_{T}\left(b_{1}\right)>80 \mathrm{GeV}$. This is motivated by the top-left panel of Fig. 2 and it immediately reduces the SM background arising from $t \bar{t} V, V \equiv W^{ \pm}, Z$ processes by almost $50 \%$.

(ii) (C-2): Given the fact that the second leading $b$-jet for the signal is not very hard, we demand that $p_{T}\left(b_{2}\right)>60 \mathrm{GeV}$. This cut also enhances the signal to background ratio to a reasonable extent.

(iii) $(C-3): p_{T}\left(b_{3}\right)$ is chosen to be $>30 \mathrm{GeV}$. 

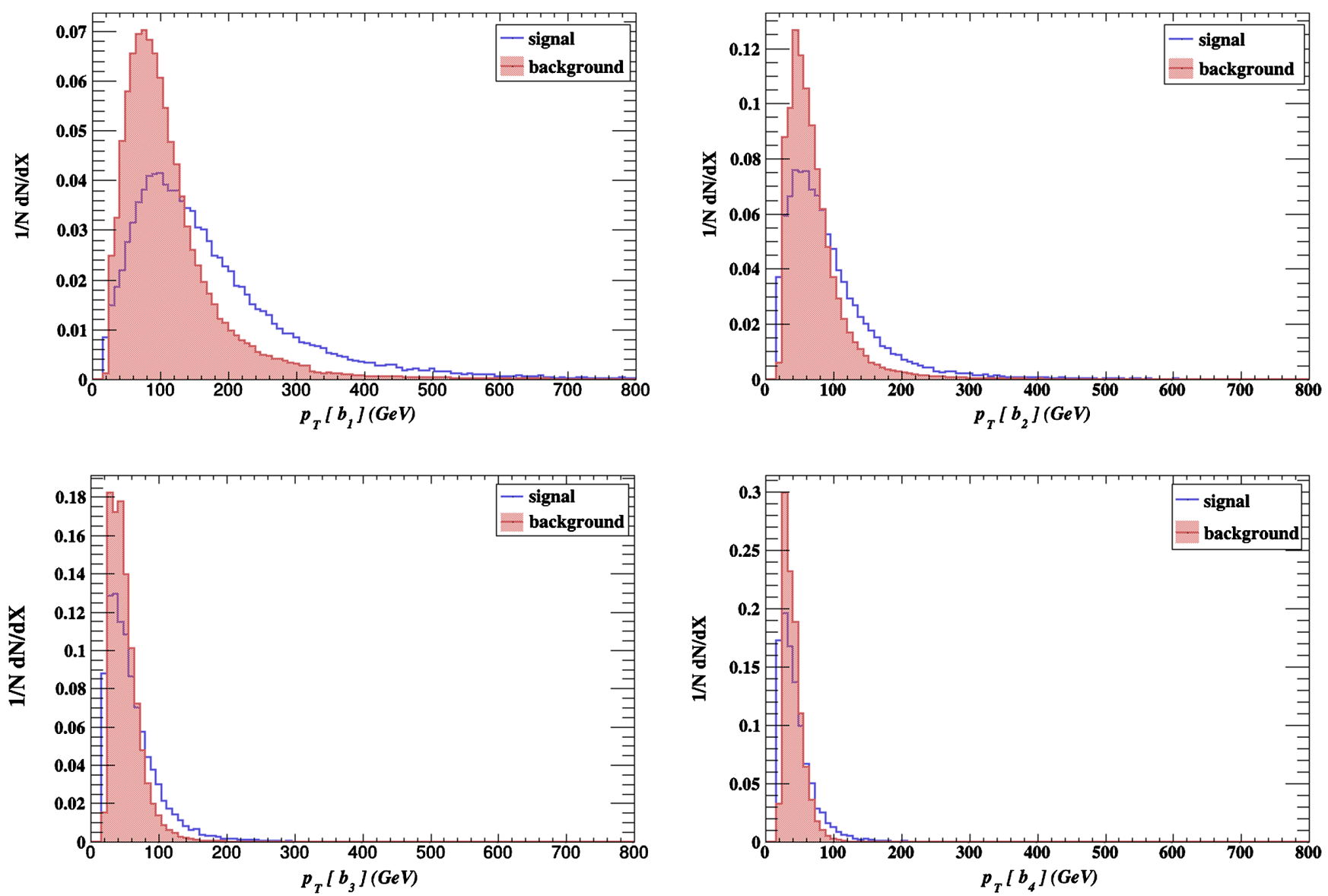

FIG. 2. Transverse momentum $\left(p_{T}\right)$ distribution (normalized) of the four leading $b$ jets for the final state $2 \ell^{ \pm} \ell^{ \pm}+4 b+\mathbb{E}_{T}$ for the benchmark BP1.
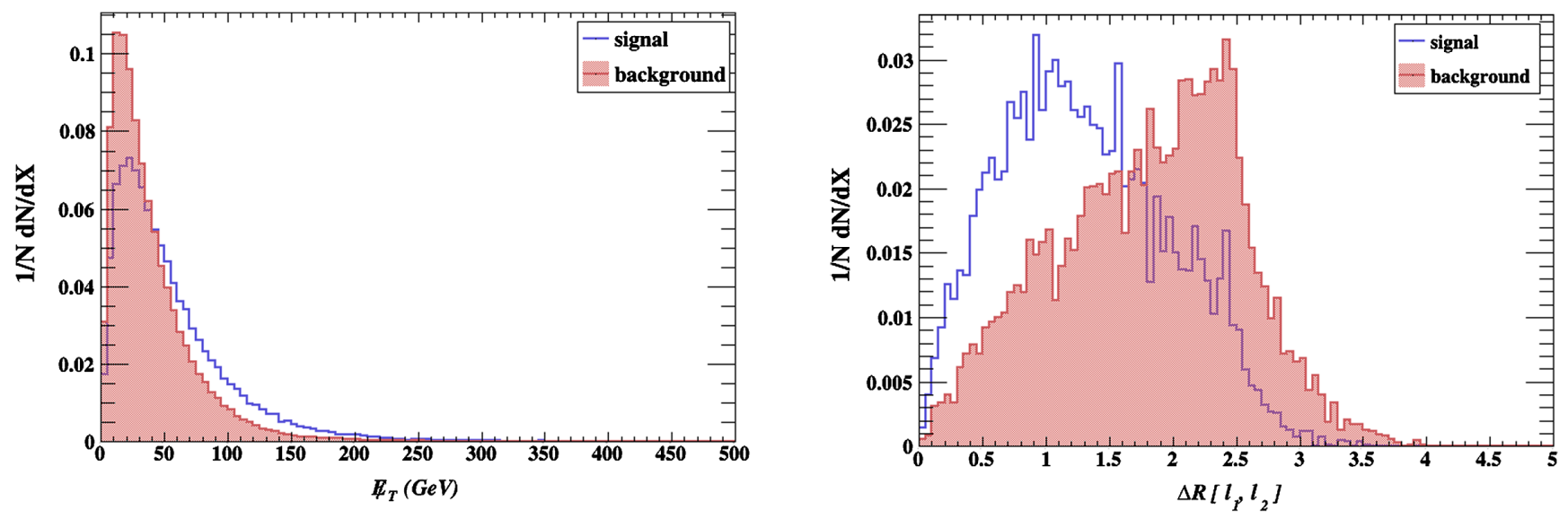

FIG. 3. The normalized missing transverse energy distribution (left panel) $\mathscr{E}_{T}$ and $\Delta R\left(\ell_{1}^{ \pm} \ell_{2}^{ \pm}\right)$distribution (normalized) (right panel) between the two same-sign leptons for the benchmark BP1.

(iv) $(C-4)$ : It is evident from the bottom-right panel of Fig. 2 that the fourth $b$ jet is very soft. So, the choice of $p_{T}\left(b_{4}\right)>20 \mathrm{GeV}$ ensures the presence of four $b$ jets in the signal.

(v) (C-5): We select two same sign leptons with $p_{T}>$ $10 \mathrm{GeV}$. (vi) (C-6): We apply a veto on any oppositely charged lepton with $p_{T}(\ell)>10 \mathrm{GeV}$.

(vii) $(C-7): \mathbb{E}_{T}>30 \mathrm{GeV}$ is imposed. This also takes care of fake $\mathscr{E}_{T}$.

(viii) (C-8): The most effective cut to reduce the SM backgrounds is the angular separation between the 
TABLE IV. Effective cross section obtained after each cut for both signal $\left(2\left(\ell^{ \pm} \ell^{ \pm}\right)+4 b+\mathbb{E}_{T}\right)$ and background and the respective significance reach at $3 \mathrm{ab}^{-1}$ integrated luminosity at $14 \mathrm{TeV}$ LHC

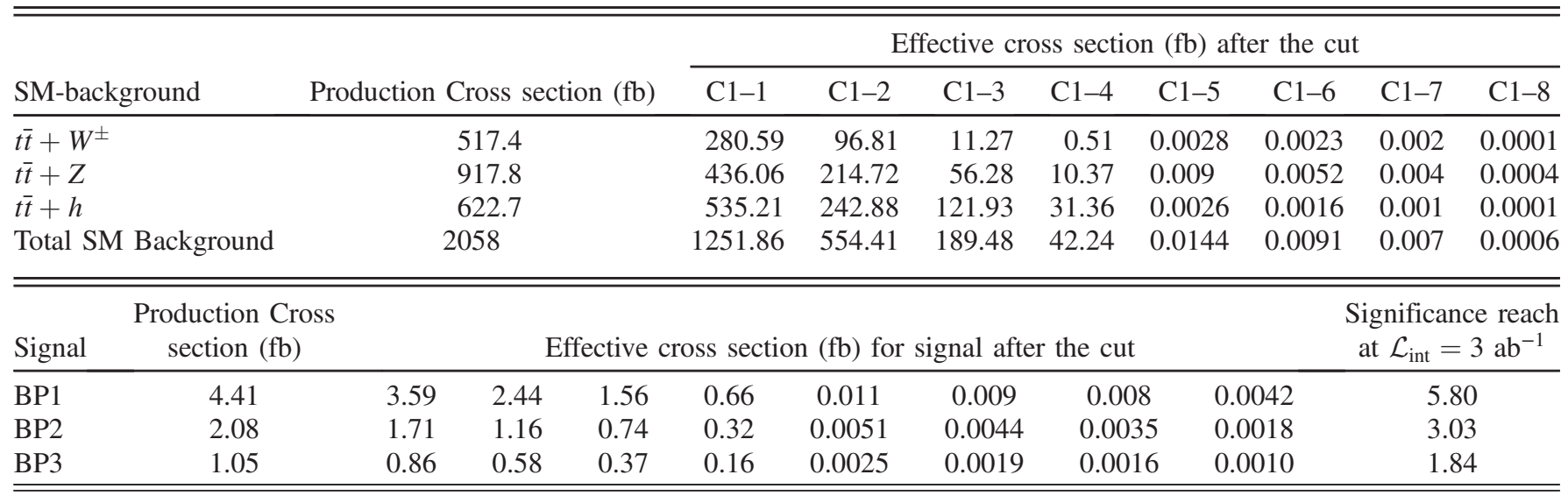

same sign dileptons. The requirement of $\Delta R\left(\ell_{1}^{ \pm} \ell_{2}^{ \pm}\right)<1.5$ considerably improves the signal to noise ratio.

With these cuts imposed, we obtain the statistical significance of the signal HE-HL LHC with $\sqrt{s}=$ $14 \mathrm{TeV}$ and also present some tentative predictions for the proposed FCC-hh with $\sqrt{s}=100 \mathrm{TeV}$.

\section{Collider search at the LHC at $\sqrt{s}=14 \mathrm{TeV}$}

Table IV contains the cut flow for $\sqrt{s}=14 \mathrm{TeV}$. The statistical significance is given by

$$
\mathcal{S}=\sqrt{2 \times\left[\left(n_{s}+n_{b}\right) \ln \left(1+\frac{n_{s}}{n_{b}}\right)-n_{s}\right]},
$$

where $n_{s}\left(n_{b}\right)$ denotes the number of signal (background) events after implementing all the cuts at a specific

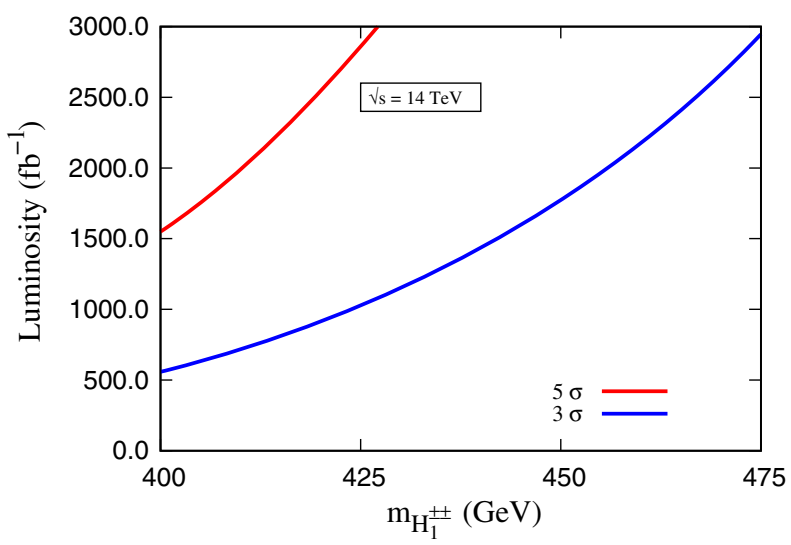

FIG. 4. Variation of the integrated luminosity $\left(\mathcal{L}_{\text {int }}\right)$ as a function of the doubly charged scalar $\operatorname{mass}\left(m_{H_{1}^{ \pm \pm}}\right)$at the $14 \mathrm{TeV}$ LHC. The red and blue lines correspond to the $\mathcal{L}_{\text {int }}$ required for the $5 \sigma$ discovery and $3 \sigma$ evidence respectively for the signal studied. luminosity. The signal significance can be seen for three benchmark points, assuming an integrated luminosity $\mathcal{L}_{\text {int }}$ of $3 \mathrm{ab}^{-1}$. We obtain the highest statistical significance $\mathcal{S}=$ 5.80 for BP1, which corresponds to $m_{H_{1}^{ \pm \pm}}=416.37 \mathrm{GeV}$ and $m_{H_{2}^{ \pm \pm}}=216.13 \mathrm{GeV}$ respectively. For the other two benchmark points with relatively heavier scalar masses, the significances are $3.03 \sigma$ and $1.84 \sigma$ respectively, which may be treated as the evidence of the two-triplet scenario.

In Fig. 4 we present the doubly charged scalar $\left(H_{1}^{ \pm \pm}\right)$ mass and the integrated luminosity $\left(\mathcal{L}_{\text {int }}\right)$ required to reach $5 \sigma$ (red solid line) and $3 \sigma$ (blue solid line) significance with $14 \mathrm{TeV}$. It is evident from this figure that "discovery" is not possible beyond $m_{H_{1}^{ \pm \pm}} \sim 430 \mathrm{GeV}$ even with $3 \mathrm{ab}^{-1}$. However, one can probe the doubly charged scalar mass up to $475 \mathrm{GeV}$ at $3 \sigma$ level.

Figure 5 shows the variation of $\mathcal{S}$ in the $m_{H_{1}^{ \pm \pm}}-m_{H_{2}^{ \pm \pm}}$ plane. $\mathcal{S}$ drops from $7 \sigma$ to $2 \sigma$ as mass of $H_{1}^{ \pm \pm}\left(H_{2}^{ \pm \pm}\right)$is

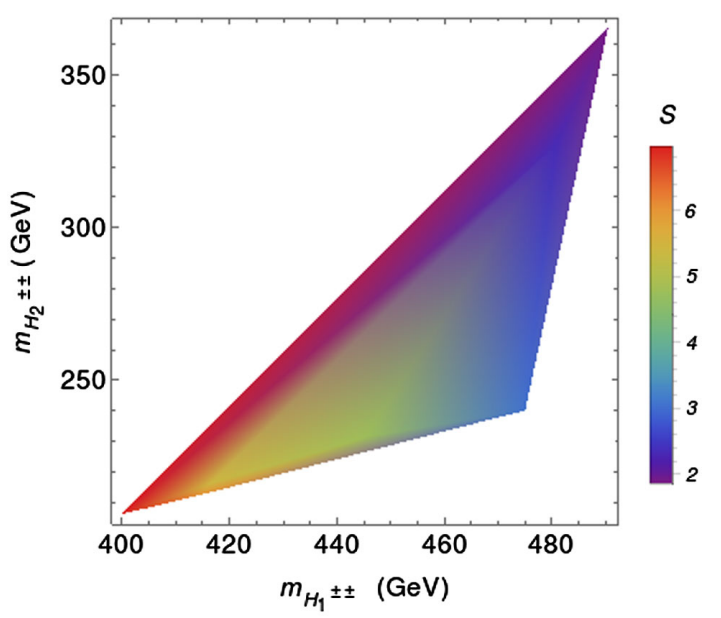

FIG. 5. Signal significance $(\mathcal{S})$ in the $m_{H_{1}^{ \pm \pm}}-m_{H_{2}^{ \pm \pm}}$plane, shown with continuously varying colour codes, for $3 \mathrm{ab}^{-1}$ integrated luminosity at $14 \mathrm{TeV}$. 
TABLE V. Effective cross section obtained after each cut for both signal $\left(2\left(\ell^{ \pm} \ell^{ \pm}\right)+4 b+\mathscr{E}_{T}\right)$ and background and the respective significance reach at $3 \mathrm{ab}^{-1}$ integrated luminosity at $100 \mathrm{TeV}$ pp collider.

\begin{tabular}{|c|c|c|c|c|c|c|c|c|c|c|c|c|}
\hline \multirow{2}{*}{\multicolumn{2}{|c|}{ SM-background }} & \multirow{2}{*}{\multicolumn{2}{|c|}{ Production Cross section (fb) }} & \multicolumn{9}{|c|}{ Effective cross section ( $\mathrm{fb}$ ) after the cut } \\
\hline & & & & \multicolumn{2}{|c|}{$\mathrm{C} 2-1$} & $\mathrm{C} 2-2$ & $\mathrm{C} 2-3$ & C2-4 & $\mathrm{C} 2-5$ & \multirow{2}{*}{ C2-6 } & $\mathrm{C} 2-7$ & C2-8 \\
\hline$\overline{t \bar{t}+W}$ & & $2.07 \times$ & $10^{4}$ & 6.27 & $\times 10^{3}$ & $1.18 \times 10^{3}$ & $1.84 \times 10^{2}$ & 10.73 & 0.10 & & 0.040 & 0.004 \\
\hline$t \bar{t}+Z$ & & $6.41 \times$ & $10^{4}$ & 3.10 & $\times 10^{4}$ & $1.12 \times 10^{4}$ & $2.77 \times 10^{3}$ & 55.09 & 0.13 & 0.12 & 0.116 & 0.009 \\
\hline $\bar{t} \bar{t}+h$ & & $3.80 \times$ & $10^{4}$ & 2.46 & $\times 10^{4}$ & $1.24 \times 10^{4}$ & $5.68 \times 10^{3}$ & $1.51 \times 10^{3}$ & 0.23 & 0.22 & 0.21 & 0.015 \\
\hline Total S & Background & $1.11 \times$ & $10^{5}$ & 6.19 & $\times 10^{4}$ & $2.48 \times 10^{4}$ & $8.63 \times 10^{3}$ & $1.57 \times 10^{3}$ & 0.46 & 0.42 & 0.366 & 0.028 \\
\hline Signal & $\begin{array}{r}\text { Production } \mathrm{C} \\
\text { section }(\mathrm{fb} \\
\end{array}$ & Cross & Effective & e $\operatorname{cros}$ & ectio & $\mathrm{n}(\mathrm{fb})$ for sig & gnal after the & cut & & & $\begin{array}{l}\text { ificance } \\
\mathcal{L}_{\text {int }}=3 \\
\end{array}$ & $\begin{array}{l}\text { reach } \\
\mathrm{ab}^{-1}\end{array}$ \\
\hline BP1 & 125.0 & 83.59 & 51.08 & 30.29 & 12.3 & 0.142 & 0.122 & 0.121 & 0.072 & & 18.21 & \\
\hline BP2 & 68.08 & 45.90 & 27.99 & 16.60 & 6.6 & 0.077 & 0.066 & 0.065 & 0.039 & & 10.80 & \\
\hline BP3 & 28.81 & 19.35 & 11.83 & 7.02 & 2.8 & 0.034 & 0.029 & 0.028 & 0.020 & & 5.94 & \\
\hline
\end{tabular}

increased from 400(206) $\mathrm{GeV}$ to $490(370) \mathrm{GeV}$ due to phase space suppression. It is worth mentioning here that, for the single-triplet case with $w \sim 1 \mathrm{GeV}$, doubly charged scalar masses up to $300 \mathrm{GeV}$ can be explored at the $5 \sigma$ level at the LHC with an integrated luminosity of $3 \mathrm{ab}^{-1}$ [41].

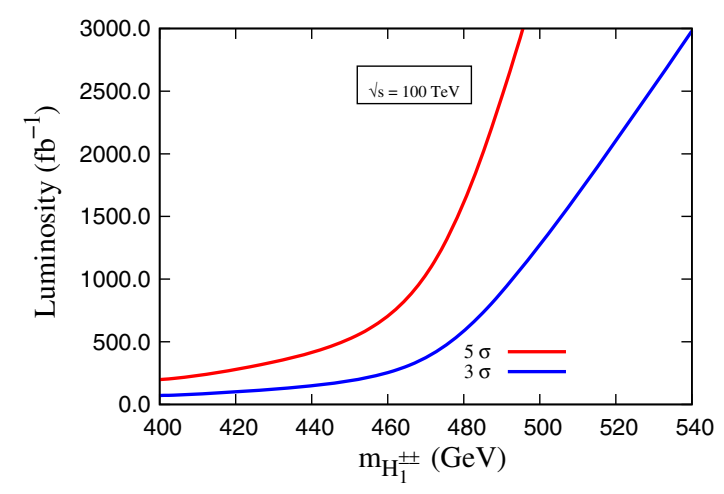

FIG. 6. Same as in Fig. 4 for the $100 \mathrm{TeV}$ FCC-hh.

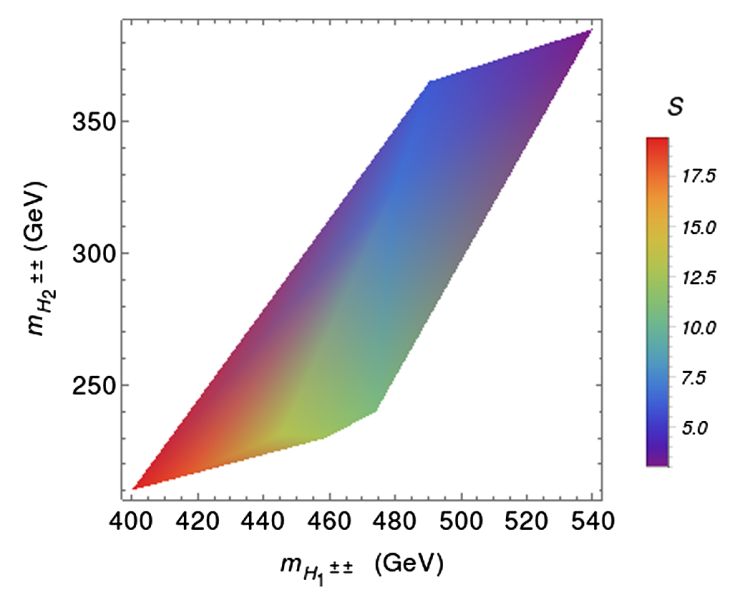

FIG. 7. Same as in Fig. 5 for the 100 TeV FCC-hh.

\section{Search at $\sqrt{s}=100$ TeV FCC-hh collider}

We next make a tentative estimate of the potential of the proposed $100 \mathrm{TeV}$ FCC-hh to investigate the two triplet scalar scenario. In our numerical analysis, we apply the set of selection cuts as those used for the $14 \mathrm{TeV}$ LHC. Since the production mode of our signal processes are electroweak processes, the NLO K-factors, too, are taken to be the same, an assumption justifiable in the light of [92]. Table V summarizes the effect of different selection cuts on the signal and the SM backgrounds for all the three benchmark points. At this energy, all of them can be probed with $\mathcal{S} \geq 5 \sigma$ at $3 \mathrm{ab}^{-1}$. A comparison with the $14 \mathrm{TeV}$ LHC shows that, while a $5 \sigma$ reach with $\left(3 \mathrm{ab}^{-1}\right)$ is possible only for BP1, a distinct improvement is foreseen for the FCC-hh. This is reflected in Fig. 6 which shows that one can probe doubly charged Higgs masses up to $495(540) \mathrm{GeV}$ at the $5 \sigma(3 \sigma)$ significance level.

The signal topology studied in this work strongly depends on $m_{H_{1}^{ \pm \pm}}, m_{H_{2}^{ \pm \pm}}$and their mass splitting. This is accentuated in Fig. 7 where we show the dependence of the signal significance on these two masses. using color-codes significance regions once more.

\section{CONCLUSION}

We have extended the Type-II seesaw model with an extra $S U(2)_{L}$ complex triplet scalar and allow a small mixing of the triplets with the SM Higgs doublet as required by electroweak precision measurements. After EWSB, one has a rather rich scalar spectrum including two each of doubly and singly charged scalars. A collider signal distinctly reflecting the presence of two triplets is identified in the form of $2 \ell^{ \pm} \ell^{ \pm}+4 b+\mathbb{E}_{T}$. This channel can arise both from $H_{1}^{ \pm \pm} \rightarrow H_{2}^{ \pm \pm} h$ and $H_{1}^{ \pm \pm} \rightarrow H_{2}^{ \pm} W^{ \pm}$following the characteristic decay modes in the case where the Yukawa couplings are too small (large triplet vev) to trigger the dominant same-sign-dilepton channels. Three 
benchmark points, consistent with all phenomenological constraints, have been used as illustration. We have estimated the potential at high luminosity LHC and a high energy pp collider $100 \mathrm{TeV}$ in identifying this signal.

We conclude after a cut-based analysis that the $5 \sigma$ discovery reach is possible with $3 \mathrm{ab}^{-1}$ luminosity at $14 \mathrm{TeV}$ LHC for the mass of the heavier doubly charged scalar up to about $430 \mathrm{GeV}$. For the proposed FCC-hh collider operating at $\sqrt{s}=100 \mathrm{TeV}$, this reach can be extended up to $495 \mathrm{GeV}$. Though we have not taken into account some experimental issues such as jet faking leptons, lepton charge misidentification, and photon conversions into lepton pairs, these effects are unlikely to affect the predictions qualitatively. Therefore, with appropriate refinement, our prediction should help in probing this scenario which is relevant in framing predictive models for neutrino masses and mixing.

\section{ACKNOWLEDGMENTS}

N. G. would like to acknowledge the Council of Scientific and Industrial Research (CSIR), Government of India for financial support. B. M. acknowledges financial support from the Department of Atomic Energy, Government of India, for the Regional Centre for Accelerator-based Particle Physics (RECAPP), Harish-Chandra Research Institute. D. K. G. and N. G. thank RECAPP for hospitality while this work was being carried out. B. M. would like to thank Indian Association for the Cultivation of Science for hospitality during the concluding part of the project.
[1] G. Aad et al. (ATLAS Collaboration), Observation of a new particle in the search for the Standard Model Higgs boson with the ATLAS detector at the LHC, Phys. Lett. B 716, 1 (2012).

[2] S. Chatrchyan et al. (CMS Collaboration), Observation of a new boson at a mass of $125 \mathrm{GeV}$ with the CMS experiment at the LHC, Phys. Lett. B 716, 30 (2012).

[3] C. W. Walter, The Super-Kamiokande experiment, arXiv: 0802.1041.

[4] M. C. Gonzalez-Garcia and M. Maltoni, Phenomenology with massive neutrinos, Phys. Rep. 460, 1 (2008).

[5] R. N. Mohapatra and A. Y. Smirnov, Neutrino mass and new physics, Annu. Rev. Nucl. Part. Sci. 56, 569 (2006).

[6] P. Minkowski, $\mu \rightarrow e \gamma$ at a rate of one out of $10^{9}$ muon decays?, Phys. Lett. 67B, 421 (1977).

[7] T. Yanagida, Horizontal symmetry and masses of neutrinos, Conf. Proc. C7902131, 95 (1979).

[8] R. N. Mohapatra and G. Senjanovic, Neutrino Mass and Spontaneous Parity Violation, Phys. Rev. Lett. 44, 912 (1980).

[9] J. Schechter and J. W. F. Valle, Neutrino masses in $S U(2) \times$ $U(1)$ theories, Phys. Rev. D 22, 2227 (1980).

[10] M. Magg and C. Wetterich, Neutrino mass problem and gauge Hierarchy, Phys. Lett. 94B, 61 (1980).

[11] T. P. Cheng and L.-F. Li, Neutrino masses, mixings and oscillations in $S U(2) \times U(1)$ models of electroweak interactions, Phys. Rev. D 22, 2860 (1980).

[12] G. Lazarides, Q. Shafi, and C. Wetterich, Proton lifetime and fermion masses in an SO(10) model, Nucl. Phys. B181, 287 (1981).

[13] R. N. Mohapatra and G. Senjanovic, Neutrino masses and mixings in gauge models with spontaneous parity violation, Phys. Rev. D 23, 165 (1981).

[14] R. Foot, H. Lew, X. G. He, and G. C. Joshi, Seesaw neutrino masses induced by a triplet of leptons, Z. Phys. C 44, 441 (1989).
[15] R. Franceschini, T. Hambye, and A. Strumia, Type-III seesaw at LHC, Phys. Rev. D 78, 033002 (2008).

[16] F. del Aguila and J. A. Aguilar-Saavedra, Distinguishing seesaw models at LHC with multi-lepton signals, Nucl. Phys. B813, 22 (2009).

[17] A. Arhrib, B. Bajc, D. K. Ghosh, T. Han, G.-Y. Huang, I. Puljak, and G. Senjanović, Collider signatures for heavy lepton triplet in type I + III seesaw, Phys. Rev. D 82, 053004 (2010).

[18] A. G. Akeroyd and M. Aoki, Single and pair production of doubly charged Higgs bosons at hadron colliders, Phys. Rev. D 72, 035011 (2005).

[19] A. G. Akeroyd and C.-W. Chiang, Doubly charged Higgs bosons and three-lepton signatures in the Higgs triplet model, Phys. Rev. D 80, 113010 (2009).

[20] A. G. Akeroyd and C.-W. Chiang, Phenomenology of large mixing for the CP-even neutral scalars of the Higgs triplet model, Phys. Rev. D 81, 115007 (2010).

[21] A. G. Akeroyd and H. Sugiyama, Production of doubly charged scalars from the decay of singly charged scalars in the Higgs triplet model, Phys. Rev. D 84, 035010 (2011).

[22] M. Lindner, M. Platscher, and F. S. Queiroz, A call for new physics: The Muon anomalous magnetic moment and lepton flavor violation, Phys. Rep. 731, 1 (2018).

[23] A. Arhrib, R. Benbrik, M. Chabab, G. Moultaka, M. C. Peyranere, L. Rahili, and J. Ramadan, The Higgs potential in the type II seesaw model, Phys. Rev. D 84, 095005 (2011).

[24] M. Aoki, S. Kanemura, M. Kikuchi, and K. Yagyu, Radiative corrections to the Higgs boson couplings in the triplet model, Phys. Rev. D 87, 015012 (2013).

[25] P. Fileviez Perez, T. Han, G.-y. Huang, T. Li, and K. Wang, Neutrino masses and the CERN LHC: Testing type II seesaw, Phys. Rev. D 78, 015018 (2008).

[26] A. Melfo, M. Nemevsek, F. Nesti, G. Senjanovic, and Y. Zhang, Type II seesaw at LHC: The roadmap, Phys. Rev. D 85, 055018 (2012). 
[27] M. Aoki, S. Kanemura, and K. Yagyu, Testing the Higgs triplet model with the mass difference at the LHC, Phys. Rev. D 85, 055007 (2012).

[28] ATLAS Collaboration, Search for doubly-charged Higgs boson production in multi-lepton final states with the ATLAS detector using proton-proton collisions at $\sqrt{s}=13 \mathrm{TeV}$, Report No. ATLAS-CONF-2017-053.

[29] T. Han, B. Mukhopadhyaya, Z. Si, and K. Wang, Pair production of doubly-charged scalars: Neutrino mass constraints and signals at the LHC, Phys. Rev. D 76, 075013 (2007).

[30] S. Chakrabarti, D. Choudhury, R. M. Godbole, and B. Mukhopadhyaya, Observing doubly charged Higgs bosons in photon-photon collisions, Phys. Lett. B 434, 347 (1998).

[31] K. Cheung and D. K. Ghosh, Triplet Higgs boson at hadron colliders, J. High Energy Phys. 11 (2002) 048.

[32] C.-W. Chiang, T. Nomura, and K. Tsumura, Search for doubly charged Higgs bosons using the same-sign diboson mode at the LHC, Phys. Rev. D 85, 095023 (2012).

[33] S. Kanemura, K. Yagyu, and H. Yokoya, First constraint on the mass of doubly-charged Higgs bosons in the same-sign diboson decay scenario at the LHC, Phys. Lett. B 726, 316 (2013).

[34] Z. Kang, J. Li, T. Li, Y. Liu, and G.-Z. Ning, Light doubly charged Higgs boson via the $W W^{*}$ channel at LHC, Eur. Phys. J. C 75, 574 (2015).

[35] S. Kanemura, M. Kikuchi, K. Yagyu, and H. Yokoya, Bounds on the mass of doubly-charged Higgs bosons in the same-sign diboson decay scenario, Phys. Rev. D 90, 115018 (2014).

[36] S. Kanemura, M. Kikuchi, H. Yokoya, and K. Yagyu, LHC Run-I constraint on the mass of doubly charged Higgs bosons in the same-sign diboson decay scenario, Prog. Theor. Exp. Phys. (2015) 051B02.

[37] C.-H. Chen and T. Nomura, Search for $\delta^{ \pm \pm}$with new decay patterns at the LHC, Phys. Rev. D 91, 035023 (2015).

[38] Z.-L. Han, R. Ding, and Y. Liao, LHC phenomenology of type II seesaw: Nondegenerate case, Phys. Rev. D 91, 093006 (2015).

[39] Z.-L. Han, R. Ding, and Y. Liao, LHC phenomenology of the type II seesaw mechanism: Observability of neutral scalars in the nondegenerate case, Phys. Rev. D 92, 033014 (2015).

[40] M. Mitra, S. Niyogi, and M. Spannowsky, Type-II seesaw model and multilepton signatures at hadron colliders, Phys. Rev. D 95, 035042 (2017).

[41] D. K. Ghosh, N. Ghosh, I. Saha, and A. Shaw, Revisiting the high-scale validity of the type II seesaw model with novel LHC signature, Phys. Rev. D 97, 115022 (2018).

[42] P. S. B. Dev and Y. Zhang, Displaced vertex signatures of doubly charged scalars in the type-II seesaw and its left-right extensions, J. High Energy Phys. 10 (2018) 199.

[43] W. Grimus, A. S. Joshipura, L. Lavoura, and M. Tanimoto, Symmetry realization of texture zeros, Eur. Phys. J. C 36, 227 (2004).

[44] A. Chaudhuri, W. Grimus, and B. Mukhopadhyaya, Doubly charged scalar decays in a type II seesaw scenario with two Higgs triplets, J. High Energy Phys. 02 (2014) 060.
[45] A. Chaudhuri and B. Mukhopadhyaya, CP-violating phase in a two Higgs triplet scenario: Some phenomenological implications, Phys. Rev. D 93, 093003 (2016).

[46] T. Golling et al., Physics at a $100 \mathrm{TeV}$ pp collider: Beyond the Standard Model phenomena, arXiv:1606.00947.

[47] J. Tang et al., Concept for a future super proton-proton collider, arXiv:1507.03224.

[48] N. Arkani-Hamed, T. Han, M. Mangano, and L.-T. Wang, Physics opportunities of a $100 \mathrm{TeV}$ protonproton collider, Phys. Rep. 652, 1 (2016).

[49] C. Patrignani et al. (Particle Data Group Collaboration), Review of particle physics, Chin. Phys. C 40, 100001 (2016).

[50] E. J. Chun, H. M. Lee, and P. Sharma, Vacuum stability, perturbativity, EWPD and Higgs-to-diphoton rate in type II seesaw models, J. High Energy Phys. 11 (2012) 106.

[51] M. Baak, J. Cúth, J. Haller, A. Hoecker, R. Kogler, K. Mönig, M. Schott, and J. Stelzer (Gfitter Group Collaboration), The global electroweak fit at NNLO and prospects for the LHC and ILC, Eur. Phys. J. C 74, 3046 (2014).

[52] P. H. Frampton, S. L. Glashow, and D. Marfatia, Zeroes of the neutrino mass matrix, Phys. Lett. B 536, 79 (2002).

[53] Z.-z. Xing, Texture zeros and Majorana phases of the neutrino mass matrix, Phys. Lett. B 530, 159 (2002).

[54] Z.-z. Xing, A full determination of the neutrino mass spectrum from two zero textures of the neutrino mass matrix, Phys. Lett. B 539, 85 (2002).

[55] M. Honda, S. Kaneko, and M. Tanimoto, Prediction and its stability in neutrino mass matrix with two zeros, J. High Energy Phys. 09 (2003) 028.

[56] W.-1. Guo and Z.-z. Xing, Calculable CP violating phases in the minimal seesaw model of leptogenesis and neutrino mixing, Phys. Lett. B 583, 163 (2004).

[57] M. Honda, S. Kaneko, and M. Tanimoto, Seesaw enhancement of bilarge mixing in two zero textures, Phys. Lett. B 593, 165 (2004).

[58] S. Goswami and A. Watanabe, Minimal seesaw textures with two heavy neutrinos, Phys. Rev. D 79, 033004 (2009).

[59] S. Choubey, W. Rodejohann, and P. Roy, Phenomenological consequences of four zero neutrino Yukawa textures, Nucl. Phys. B808, 272 (2009).

[60] S. Goswami, S. Khan, and W. Rodejohann, Minimal textures in seesaw mass matrices and their low and high energy phenomenology, Phys. Lett. B 680, 255 (2009).

[61] H. Fritzsch, Z.-Z. Xing, and S. Zhou, Two-zero textures of the Majorana neutrino mass matrix and current experimental tests, J. High Energy Phys. 09 (2011) 083.

[62] M. Ghosh, S. Goswami, and S. Gupta, Two zero mass matrices and sterile neutrinos, J. High Energy Phys. 04 (2013) 103.

[63] J. Liao, D. Marfatia, and K. Whisnant, Neutrino seesaw mechanism with texture zeros, Nucl. Phys. B900, 449 (2015).

[64] T. Kitabayashi and M. Yasu, Formulas for flavor neutrino masses and their application to texture two zeros, Phys. Rev. D 93, 053012 (2016).

[65] J. M. Lamprea and E. Peinado, Seesaw scale discrete dark matter and two-zero texture Majorana neutrino mass matrices, Phys. Rev. D 94, 055007 (2016). 
[66] W. Grimus and L. Lavoura, On a model with two zeros in the neutrino mass matrix, J. Phys. G 31, 693 (2005).

[67] J. Beringer et al. (Particle Data Group Collaboration), Review of particle physics (RPP), Phys. Rev. D 86, 010001 (2012).

[68] G. L. Fogli, E. Lisi, A. Marrone, D. Montanino, A. Palazzo, and A. M. Rotunno, Global analysis of neutrino masses, mixings and phases: Entering the era of leptonic CP violation searches, Phys. Rev. D 86, 013012 (2012).

[69] F. P. An et al. (Daya Bay Collaboration), Observation of Electron-Antineutrino Disappearance at Daya Bay, Phys. Rev. Lett. 108, 171803 (2012).

[70] J. K. Ahn et al. (RENO Collaboration), Observation of Reactor Electron Antineutrino Disappearance in the RENO Experiment, Phys. Rev. Lett. 108, 191802 (2012).

[71] F. Arbabifar, S. Bahrami, and M. Frank, Neutral Higgs bosons in the Higgs triplet model with nontrivial mixing, Phys. Rev. D 87, 015020 (2013).

[72] S. Kanemura and K. Yagyu, Radiative corrections to electroweak parameters in the Higgs triplet model and implication with the recent Higgs boson searches, Phys. Rev. D 85, 115009 (2012).

[73] A. G. Akeroyd and S. Moretti, Enhancement of H to gamma gamma from doubly charged scalars in the Higgs triplet model, Phys. Rev. D 86, 035015 (2012).

[74] P. S. Bhupal Dev, D. K. Ghosh, N. Okada, and I. Saha, $125 \mathrm{GeV}$ Higgs boson and the type-II seesaw model, J. High Energy Phys. 03 (2013) 150.

[75] D. Das and A. Santamaria, Updated scalar sector constraints in the Higgs triplet model, Phys. Rev. D 94, 015015 (2016).

[76] A. M. Sirunyan et al. (CMS Collaboration), Measurements of Higgs boson properties in the diphoton decay channel in proton-proton collisions at $\sqrt{s}=13 \mathrm{TeV}, \mathrm{J}$. High Energy Phys. 11 (2018) 185.

[77] J. Alwall, R. Frederix, S. Frixione, V. Hirschi, F. Maltoni, O. Mattelaer, H.-S. Shao, T. Stelzer, P. Torrielli, and M. Zaro, The automated computation of tree-level and next-toleading order differential cross sections, and their matching to parton shower simulations, J. High Energy Phys. 07 (2014) 079.

[78] A. Alloul, N. D. Christensen, C. Degrande, C. Duhr, and B. Fuks, FeynRules 2.0: A complete toolbox for treelevel phenomenology, Comput. Phys. Commun. 185, 2250 (2014).
[79] J. Pumplin, D. R. Stump, J. Huston, H. L. Lai, P. M. Nadolsky, and W. K. Tung, New generation of parton distributions with uncertainties from global QCD analysis, J. High Energy Phys. 07 (2002) 012.

[80] ATLAS Collaboration, Search for doubly-charged Higgs bosons in same-charge electron pair final states using proton-proton collisions at $\sqrt{s}=13 \mathrm{TeV}$ with the ATLAS detector, Report No. ATLAS-CONF-2016-051.

[81] J. M. Campbell and R. K. Ellis, $t \bar{t} W^{+-}$production and decay at NLO, J. High Energy Phys. 07 (2012) 052

[82] A. Lazopoulos, T. McElmurry, K. Melnikov, and F. Petriello, Next-to-leading order QCD corrections to $t \bar{t} Z$ production at the LHC, Phys. Lett. B 666, 62 (2008).

[83] S. Frixione, V. Hirschi, D. Pagani, H. S. Shao, and M. Zaro, Electroweak and QCD corrections to top-pair hadroproduction in association with heavy bosons, J. High Energy Phys. 06 (2015) 184.

[84] T. Sjostrand, S. Mrenna, and P.Z. Skands, PYTHIA 6.4 physics and manual, J. High Energy Phys. 05 (2006) 026.

[85] J. de Favereau, C. Delaere, P. Demin, A. Giammanco, V. Lematre, A. Mertens, and M. Selvaggi (DELPHES 3 Collaboration), DELPHES 3: A modular framework for fast simulation of a generic collider experiment, J. High Energy Phys. 02 (2014) 057.

[86] M. Cacciari, G. P. Salam, and G. Soyez, The anti- $k(t)$ jet clustering algorithm, J. High Energy Phys. 04 (2008) 063.

[87] E. Conte, B. Fuks, and G. Serret, MadAnalysis 5: A userfriendly framework for collider phenomenology, Comput. Phys. Commun. 184, 222 (2013).

[88] ATLAS Collaboration, Calibration of the performance of $b$-tagging for $c$ and light-flavour jets in the 2012 ATLAS data, Report No. ATLAS-CONF-2014-046.

[89] ATLAS Collaboration, Measurement of the $W^{+} W^{-}$production cross section in $p p$ collisions at a centre-of-mass energy of $\sqrt{s}=13 \mathrm{TeV}$ with the ATLAS experiment, Report No. ATLAS-CONF-2016-090.

[90] ATLAS Collaboration, Electron efficiency measurements with the ATLAS detector using the 2015 LHC protonproton collision data, Report No. ATLAS-CONF-2016-024.

[91] G. Aad et al. (ATLAS Collaboration), Muon reconstruction performance of the ATLAS detector in protonproton collision data at $\sqrt{s}=13 \mathrm{TeV}$, Eur. Phys. J. C 76, 292 (2016).

[92] M. L. Mangano et al., Physics at a $100 \mathrm{TeV}$ pp collider: Standard model processes, arXiv:1607.01831. 